\title{
The Research on Tibetan Compound Consonants of Propositional Nasal [n] in Xiahe Dialect by Means of Electropalatograph
}

\author{
Dawa Pengcuo ${ }^{a}$, Han $\mathrm{Wu}^{\mathrm{b}}$
}

\author{
Key Lab of China's National Linguistic Information Technology of Ministry of Education, Northwest \\ University for Nationalities, Lanzhou 730000, China \\ adawapengcuo@163.com, bwu2006han@126.com
}

Keywords: Dynamic electropalatograph, Tibetan, Xiahe, compound consonants.

\begin{abstract}
In our study we focus on Xiahe dialect which is one of the most representative Ando dialect in Tibetan, and research the compound consonants of [nd], [ndz], [ndz] and [nd $\mathbf{p}_{\text {] }}$ by dynamic electropalatograph, whose propositional nasal is [n]. In this paper, the frame of each compound consonant is analyzed, which is the biggest contact area of tongue and palatal of main consonant; and two parameters , contact anteriority (CA) and contact centrality(CC) are extracted. The influence of propositional nasal[n] on the main consonant is studied, comparing the differences between main consonants of compound consonants and single consonants which is the same place of articulation. From this study, we find that the position of the main consonant is affected by the propositional nasal in different degree and it is close to the direction of the preposition consonant [n].
\end{abstract}

\section{Introduction}

The research of traditional phonetics on consonant is static, it is mainly relied on people's perception to describe and study consonant pronunciation parts and method. Dynamic electropalatography can more objectively and accurately record and describe voice by recording the tongue and palate contact state in pronunciation, the traditional phonetics on phonetic interpretation and description are deepened. The main advantage of dynamic electropalatography lies in its "observe language in real time" function, and synchronously, intuitively, continuously and objectively present the tongue and palate contact state in the whole pronunciation process in front of people, it is a kind of intact and non-invasive detection means. At present, the research on dynamic palate position has been extended to the UK, France, Germany, Japan, Netherlands, Spain, Hungary, Finland, and Korea more than 20 languages. In recent years, the domestic introduced dynamic electropalatography, the speech production mechanism, phonological features, coarticulation, language teaching, language barrier and speech rehabilitation training research are carried out, dynamic palate position research of Putonghua, Shanghai dialect, Mongolian have made a lot of achievements [1] [2] [3].

The Tibetan is divided into Ando dialect, Weizang dialect and Kang dialect. Ando dialect belongs to dialect that retains the ancient Tibetan phenomenon more in Tibetan three major dialects, syllable only has habitual tone, and there are complex initials systems of compound consonant. The research of compound consonant has positive significance in exploring the history of the Tibetan pronunciation and Tibetan dialect, and even the language evolution. Domestic research on Tibetan compound consonant mainly concentrated on the phonology and the acoustics level, Kong Jiangping make acoustic research of Tibetan consonants [4]. This article chooses the most representative Xiahe language in Ando dialect words as the research object, and dynamic palate research of compound consonant are carried out, explore difference between single consonants and compound consonant in the same places of articulation but belong to different pronunciation methods.

\section{Research Method}

According to the speakers' mouth shape, electronic false palate is made, whether the tongue contact hard palate or not in pronunciation when power on, contact position of tongue and palate is 
displayed on the screen (100 tongue and palate contact photo are shot within 1 second), and give visual feedback to researchers. In the research of single consonants, frame of biggest contact place of palate and tongue in consonants is selected as the research object. Compound consonant adopts the same research method with single consonants, the biggest frame in the tongue and palate contact area of the primary consonants in the compound consonant before release are mainly choose to studied, extract Contact Anteriority in Fontdevila design (Contact Anteriority, abbreviated as CA) and Contact Centrality (Contact Centrality, abbreviated as CC) [5] two parameters. The greatest significance of two indexes is that although the index value overall increases with increase of the total contact places, but the values most significantly does not influence the absolute increase of contact places, but the distribution features of the contact place. As long as contact place is not beyond a row or column, the index value will be in a fixed range.

\subsection{Contact Anteriority}

CA reflect the front degree of contact part from the angle of horizontal lines, the range is between $0 \sim 1$, 0 means empty contact, 1 means full contact, the closer to 1 the CA, tongue and palate contact are more front, calculating formula as shown in the following:

$\mathrm{CA}=\log [[1 *(\mathrm{R} 8 / 8)+9 *(\mathrm{R} 7 / 8)+81 *(\mathrm{R} 6 / 8)+729 *(\mathrm{R} 5 / 8)+6567 *(\mathrm{R} 4 / 8)+59049 *(\mathrm{R} 3 / 8)+531441 *(\mathrm{R} 2$ $/ 8)+3587227 *(\mathrm{R} 1 / 6)]+1] / \log [(1+9+81+729+6567+59049+531441+3587227)+1]$

\subsection{Contact Centrality}

CC make longitudinal line as basis and reflect the concentrated degree of contact place from both sides to center, the range is between $0 \sim 1,0$ means empty contact, 1 mean full contact, the closer to 1 the $\mathrm{CC}$, the tongue and palate contact are more concentrated, calculating formula as shown in the following:

$$
\mathrm{CC}=[\log [1 *(\mathrm{Cl} / 14)+17 *(\mathrm{C} 2 / 16)+289 *(\mathrm{C} 3 / 16)+4913 *(\mathrm{C} 4 / 16)+1]] / \log (5220+1)
$$

\section{Dynamic Palate Position Analysis of Compound Consonant}

The compound consonant exists in Tibetan Xiahe language, with bidentate and triad compound consonant, totally twenty-nine, the bidentate compound consonant are most [s], [h], [h ] and [n]. Propositional consonant of bidentate compound consonant are [s], [h], [h] and [n], Propositional consonant are [n]: [nd], [ndz], [ndz], and [nd;], etc.

\subsection{Compound Consonant [nd]}

The places of articulation of single consonants [d] and [t] are exactly same, they are a pair of consonant with opposing voiceless and voiced sound in pronunciation methods, [t] its places of articulation are very forward, the tongue and palate contact incisor, the front gum area, covered gum ridge belong to the tongue tip alveolar, gum in pronunciation, contact anteriority and contact centrality are higher. [nd] added nasal sound $\mathrm{n}$ pronunciation action before consonants [d]. Fig.1 is palate position of [nd] and [t] collocating different vowels, table 1 are the corresponding CA and CC parameter values.
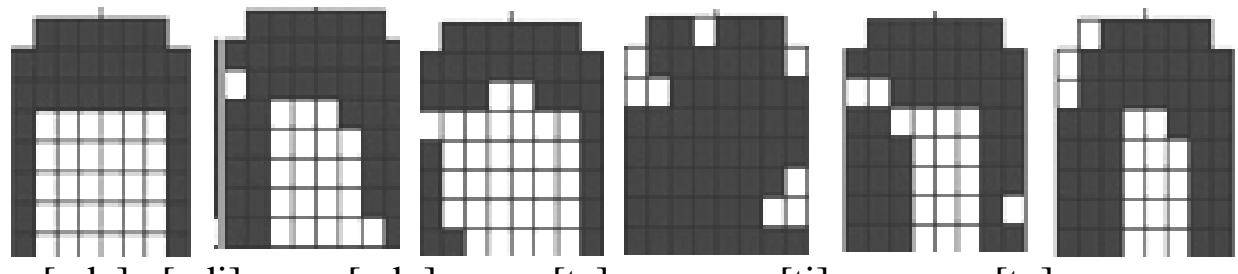

[nda] [ndi]

[ndu]

[ta]

[ti]

[tu]

Fig. 1 Dynamic palate position graph of [nd] and [t]

Table 1 CA and CC parameter table of compound consonant [nd] and single consonant [t]

\begin{tabular}{ccccccc}
\hline \multirow{2}{*}{ IPA } & \multicolumn{3}{c}{ nd } & & \multicolumn{3}{c}{$\mathrm{t}$} \\
\cline { 2 - 7 } & nda & ndi & ndu & ta & ti & tu \\
\hline CA & 1 & 1 & 1 & 0.987 & 1 & 0.988 \\
CC & 0.885 & 0.887 & 0.842 & 0.993 & 0.881 & 0.892 \\
\hline
\end{tabular}


It can be concluded that from Table 1, in the consonant cluster [nd], pronunciation action of nasal sound [n] does not have a big impact on anteroposterior position of [d] pronunciation posture. In tongue and palate contact most large area moment of consonant cluster [nd], CC and CA index is also very big, the CA index is close to 1 , pronunciation position is fairly front, CC parameter is also very high, it is showed that when pronounce [nd] this consonants, the lingual tightening place is high, position of pronunciation is in front, the pronunciation posture of the syllable is very strict, it is not easily affected by coarticulation.

\subsection{Compound Consonant (NDZ)}

Single consonants (dz) and (ts) is a pair consonant, whose places of articulation are the same, voiceless sound are opposing to voiced sound in pronunciation methods, [ndz] add pronunciation action of nasal sound [n] before the consonants (dz). Fig.2 is dynamic palate position graph of [ndz] and [ts] collocating different vowels, Table 2 is the corresponding CA and CC parameter values.

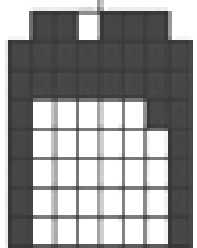

[ndza]

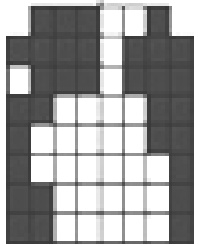

[ndzi]

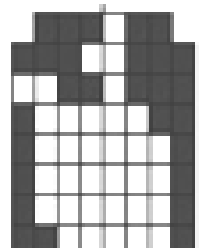

[ndzu]

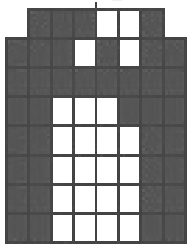

[tsa]

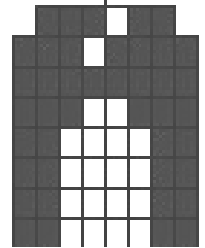

[tsi]

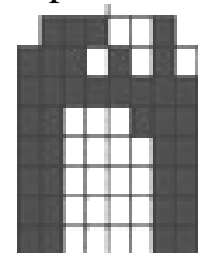

[tsu]

Fig. 2 Dynamic palate position of [ndz] and [ts]

Table 2 CA and CC parameter table of consonant cluster [ndz] and single consonant [ts]

\begin{tabular}{ccccccc}
\hline \multirow{2}{*}{ IPA } & \multicolumn{3}{c}{ ndz } & \multicolumn{3}{c}{ ts } \\
\cline { 2 - 7 } & ndza & ndzi & ndzu & tsa & tsi & tsu \\
\hline CA & 0.99 & 0.976 & 0.987 & 0.925 & 0.918 & 0.903 \\
CC & 0.866 & 0.81 & 0.77 & 0.841 & 0.845 & 0.841 \\
\hline
\end{tabular}

It can be concluded that from Table 2, the CA and CC of consonant cluster (ndz) overall are bigger than the index of single consonants (ts), especially CA is significantly high. The place of articulation of single consonant pronunciation (ts) is in front of the palate, but place of articulation of consonants [n] is more in front, when following the same vowel, CA and CC of [dz] in consonant cluster [ndz] generally increase, it is clear that place of articulation is affected by propositional consonant [n], place of articulation is close to [n].

\subsection{Compound Consonant [ndz]}

Single consonants [dz] and [ts] is a pair of consonant, whose places of articulation are exactly same, voiceless sound are opposing to voiced sound in pronunciation methods, [ndz, have pronunciation action of nasal sound [n] in advance before consonant [dz, pronunciation action. There is no words combined [ndz, ] and vowels [u]. Fig.3 is dynamic palate position graph of [ndz] and [tș] collocating different vowels, Table 3 is the corresponding CA and CC parameter values.


Fig. 3 Dynamic palate position of [ndz] and [ts]

Table 3 CA and CC parameter table of consonant cluster [ndz] and single consonant [tș]

\begin{tabular}{cccccc}
\hline \multirow{2}{*}{ IPA } & \multicolumn{2}{c}{ ndz } & \multicolumn{3}{c}{ tș } \\
\cline { 2 - 6 } & ndz_a & ndz̧, & tșa & tși & tșu \\
\hline CA & 0.81 & 0.832 & 0.762 & 0.681 & 0.686 \\
CC & 0.69 & 0.704 & 0.84 & 0.456 & 0.381 \\
\hline
\end{tabular}


It can be concluded from Table 3, the single consonants [tș] and [ndz] can be reach third row position of palate position paragraph, difference of tongue and palate contact condition is not big. But it can be seen from the numerical when following the same vowel, [ndz] CA and CC value are significantly bigger than [ts]. [dz] in [ndz] is affected by the prepositional consonant [n], place of articulation is close to prepositional consonants [n] to a certain extent [n].

\subsection{Compound Consonant [nd ]}

Single consonants $\left[\mathrm{d}_{+}\right]$and $\left[\mathrm{t}_{\boldsymbol{c}}\right]$ is a pair of consonant, whose places of articulation are exactly same, voiceless sound are opposing to voiced sound in pronunciation methods, [ nњ ] have pronunciation action of nasal sound [n] in advance before consonant [\$]]pronunciation action. Fig.4 is dynamic palate position graph of [nd;] and [tc] collocating different vowels, Table 4 is the corresponding CA and CC parameter values.

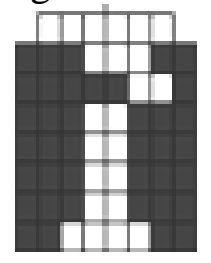

[nca]

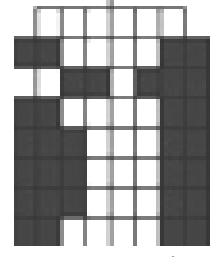

[ndii]

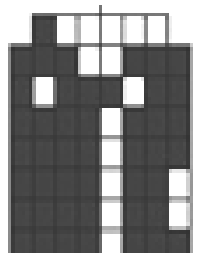

[ndiu]

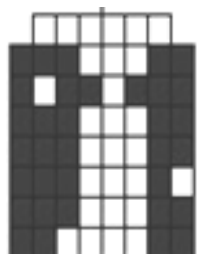

[tca]

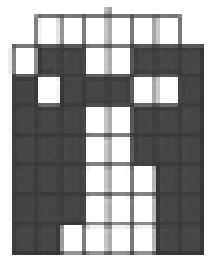

[tci]

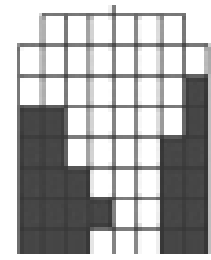

[tcu]

Fig. 4 Dynamic palate position of [nd $]$ and [tc]

Table 4 CA and CC parameter table of consonant cluster [nd ] and single consonant [tc]

\begin{tabular}{|c|c|c|c|c|c|c|}
\hline \multirow{2}{*}{ IPA } & \multicolumn{3}{|c|}{$\mathrm{nd}$} & \multicolumn{3}{|c|}{ tc } \\
\hline & ndza & ndqi & ndiu & tca & tci & tcu \\
\hline CA & 0.843 & 0.829 & 0.709 & 0.843 & 0.842 & 0.606 \\
\hline CC & 0.782 & 0.704 & 0.909 & 0.713 & 0.78 & 0.692 \\
\hline
\end{tabular}

It can be concluded from Table 4, when nd, followed by vowels [a] and [i], compared with CA and CC value single consonant [tc], change is small, when pronounce single consonant [tc], the limit of lingual surface is high, the influence of coarticulation is small. The pronunciation action of front nasal sound $[n]$ is the pronunciation action of the nasal terminal $n$, when pronounce the posture of the tongue tip can point to the gum, and don't need arrive, at the same time, the soft palate lower, open nasal passages. When following the vowels [u], CC is obviously bigger than single consonants [tc],


influence of the vowels [u], the longitudinal tongue palatal area increases.

\section{Conclusion}

This paper analyze consonant cluster of prepositional nasal sound in Xiahe language by using the electronic palate technology, and study tongue and palate contact state of the consonant cluster in pronunciation, it is more objectively and accurately record and describe physiological pronunciation process of consonant cluster, revealing the tongue and palate contact state of consonants and coarticulation phenomenon in syllable interior, and comparing with single consonants, study the change condition of tongue position of second consonants affected by the prepositional consonants. To make a more scientific and accurate research on Tibetan Ando dialect, provide the basic data for pronunciation and speech production mechanism of Tibetan Ando dialect to, and provide research of voice and vice linguistic behavior with help as well.

\section{Acknowledgement}

Thanks for Regional Fund (61262052) in the National Natural Foundation of China. 


\section{References}

[1] Bao Huaiqiao, Zheng yuling, The Research of Dynamic Electropalatograph for Mandarin, Journal of Liberal Arts of Nanjing Normal University .2011, (03), pp.1-11.

[2] Ping Yueling. The Research Of Dynamic Electropalatograph for Plosive and Affricate in the Research of Dynamic Electropalatograph for Plosive and Affricate in Shanghainese, The Sixth Modern Phonetics Conference. Tianjing, 2003, p. 31-36.

[3] Hasi Qimuge, Zheng Yuling, Hu He, etc... The Database of Dynamic Electropalatograph for Mongolian, The Ninth Phonetics Conference of Chinese. Tianjin 2010. pp. 41-48

[4] Kong Jiangping, The Acoustic Analysis of Double Fricative Initials in Daofu Tibetan. Minority Languages of China. Vol. 1991, No. 3, p. 59-64.

[5] Fontdevila. J, Pallares, M.D \& Reeasens, D. The Contact Index Method of Electropalatogrpahic Data Reduetion. Journal of Phonetics, (1994) 22, 141-154. 